\title{
Elbow Imaging with an Emphasis on MRI
}

\author{
Mark W. Anderson and Christine B. Chung
}

\section{Learning Objectives}

- To review challenges in MR imaging of the elbow and identify strategies to optimize imaging protocols.

- To review osseous and soft tissue anatomy of the elbow.

- To demonstrate the MR imaging appearance of commonly encountered pathology of the elbow.

\subsection{Introduction}

The elbow is a complex joint made up of three separate articulations within a common capsule. The proximal ulna articulates with the trochlea and functions as a hinge joint, while the proximal radioulnar joint provides for rotational movement of the forearm. The radiocapitellar joint allows for both hinge and rotational movements. Together, these allow for flexion and extension of the arm and, in conjunction with the distal radioulnar joint at the wrist, pronation and supination as well.

This article will discuss the normal anatomy of the elbow and the most common types of elbow pathology as well as their appearances on various imaging studies, with an emphasis on MR imaging.

M. W. Anderson $(\bowtie)$

Department of Radiology, University of Virginia,

Charlottesville, VA, USA

e-mail: mwa3a@virginia.edu

C. B. Chung

Department of Radiology, University of California, San Diego, La Jolla, CA, USA

e-mail: cbchung@ucsd.edu

\subsection{MR Technique}

Challenges associated with elbow MRI include the complex anatomy of this relatively small joint, as well as technical considerations related to MR acquisition. If the patient is scanned in a supine position with arms extended along the sides, this may result in some degradation of image quality given the off-center position within the magnet. Images can be acquired with the patient prone and the arm extended over the head (superman position), placing the region of interest closer to the magnet isocenter; however patient discomfort can lead to increased motion artifact.

MR imaging studies of the elbow are commonly performed at field strengths of $1.5 \mathrm{~T}$ or higher. Imaging at $3.0 \mathrm{~T}$ is increasingly common, with ultra-high field systems $(7.0 \mathrm{~T})$ deployed in the research setting [1]. Higher field strength and dedicated coils for elbow MR provide much needed improvements in signal-to-noise ratio (SNR), resulting in improved image resolution. Surface coils are essential for the acquisition of optimal MR images.

Imaging protocols should cover the joint in multiple planes and with multiple different tissue contrasts [2]. The coronal oblique imaging plane is oriented parallel to the humeral epicondylar axis as seen in the axial plane, and sagittal sequences are oriented perpendicular to that axis. Axial images should always extend distal to the radial tuberosity.

Suggested protocol parameters for elbow MR imaging include a field of view (FOV) of $12-15 \mathrm{~cm}$. Slice thickness from 2 to $3 \mathrm{~mm}$ is generally accepted with a matrix of $256 \times 256$ to $512 \times 512$. Conventional MR protocols include some combination of non-fat-suppressed T1- or PD-weighted, fat-suppressed PD- or T2-weighted, or short tau inversion recovery (STIR) sequences.

Direct MR arthrography is an alternative to conventional MR imaging that provides joint distention for better delineation and visualization of structures. Its primary application in clinical practice is for identification and characterization of intraarticular bodies and osteochondral lesions [3]. The optimum 
gadolinium concentration for maximal signal-to-noise ratios at 1.5-3.0 T ranges from 0.7 to $3.4 \mathrm{mmol} / \mathrm{L}$ [4]. The capacity of the joint for purposes of MR arthrography is 5-10 mL.

Although fat-suppressed T1-, PD-, and T2-weighted sequences are typically used, non-fat-suppressed sequences should be included if visualization of fat is desired.

\subsection{Bones and Cartilage}

\subsubsection{Normal Anatomy}

The humerus, ulna, and radius all contribute to the elbow joint [5]. Two epicondyles project from the distal humerus. The larger medial epicondyle is the site of attachment of the common flexor/pronator tendon as well as the ulnar collateral ligament. The lateral epicondyle gives rise to the common extensor/supinator tendon and the extensor carpi ulnaris tendon. The radial collateral and lateral ulnar collateral ligaments also arise from the lateral epicondyle. The distal articular surface of the humerus is divided into the trough-like trochlea that articulates with the proximal ulna and the rounded capitellum that articulates with the radial head. Dorsally, the deep, olecranon fossa of the humerus accepts the tip of the olecranon when the elbow is extended, while the smaller coronoid fossa along the ventral aspect of the humerus receives the coronoid process in flexion. Prominent extrasynovial fat pads are present within both fossae.

The trochlear notch of the ulna forms a hinge joint with the trochlea. The radial notch of the ulna lies along the lateral aspect of the coronoid process and articulates with the radial head to form the proximal radioulnar joint. The slightly concave radial head articulates with the capitellum. The radial tuberosity along the medial aspect of the proximal radius provides the attachment site for the distal biceps tendon.

The articular cartilage of the elbow is so thin that it's often difficult to evaluate with MR imaging. It's also important to recognize that the articular cartilage of the capitellum is found along its anterior convexity only, and not along its irregular, posterior non-articular aspect.

\subsubsection{Bones and Cartilage: Potential Pitfalls}

With the elbow extended, the border between the articular cartilage of the capitellum and its irregular non-articular posterior surface may mimic an osteochondral lesion on coronal MR images. This pitfall can be easily avoided by crossreferencing the level of the coronal scan with a sagittal image (Fig. 3.1). Another osseous variant is a transverse bony ridge in the mid-trochlear notch that may mimic a loose body or osteophyte on sagittal images (Fig. 3.2).
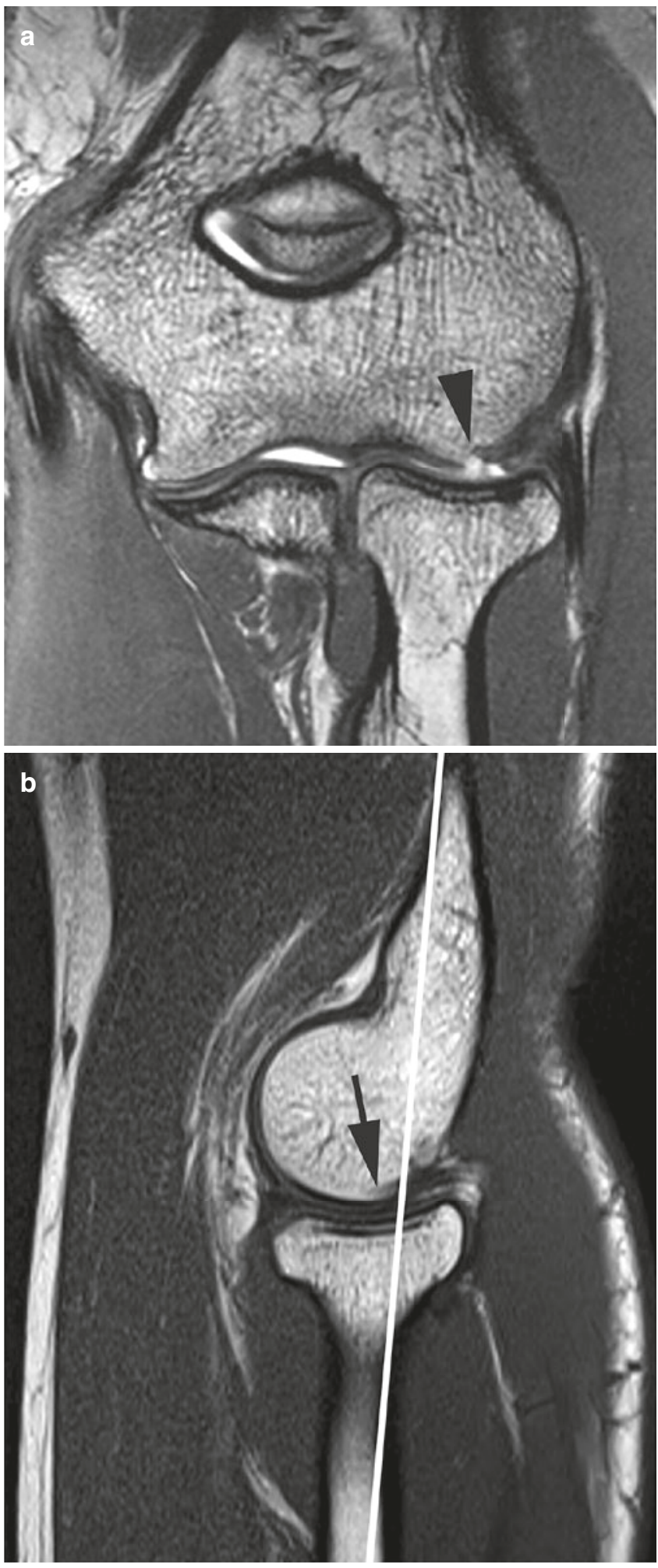

Fig. 3.1 Pseudodefect of the capitellum. (a) Coronal gradient echo T2*weighted image shows an apparent osteochondral lesion along the surface of the capitellum (arrowhead). (b) Correlative sagittal T1-weighted image reveals that the coronal slice (white line) lies just posterior margin of the articular surface (arrow) 


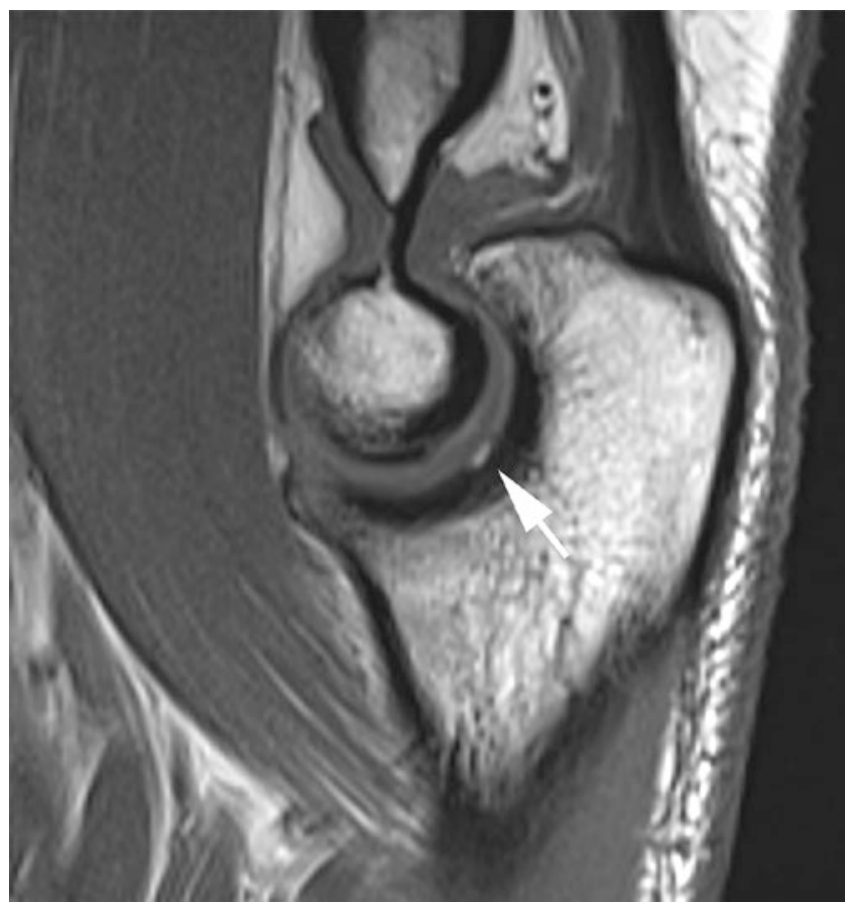

Fig. 3.2 Transverse trochlear ridge. Sagittal T1-weighted image demonstrates the normal transverse trochlear ridge (arrow) of the ulna, the anatomic landmark between the olecranon and coronoid

\subsubsection{Bones: Pathology}

\subsubsection{Acute Trauma}

In a patient presenting with elbow pain after an acute injury, often the only radiographic finding is elevation of the posterior and/or anterior fat pads indicating an effusion. Several studies have demonstrated a high incidence of associated osseous contusions or fractures in these patients which are well demonstrated with MR imaging [6]. A bone contusion is most easily identified by its edema-like signal intensity on fat-saturated T2-weighted images. In the case of a fracture, a linear component may be seen within the edema on $\mathrm{T} 2 \mathrm{~W}$ images or may be better demonstrated as a low signal intensity line on a T1-weighted image (Fig. 3.3). In the setting of a radiographically apparent fracture, MR imaging is able to assess any associated ligament or cartilage abnormalities as well. Contusions or fractures involving the radial head and posterior capitellum are commonly seen after a posterior dislocation and should prompt close inspection of the collateral ligaments which are often concurrently disrupted [7].

\subsubsection{Stress Fractures}

Stress fractures occur much less commonly in the upper than the lower extremities. In the elbow stress fractures involve the olecranon in $98 \%$ of cases [8]. The mechanism of injury is thought to be related to chronic traction of the triceps
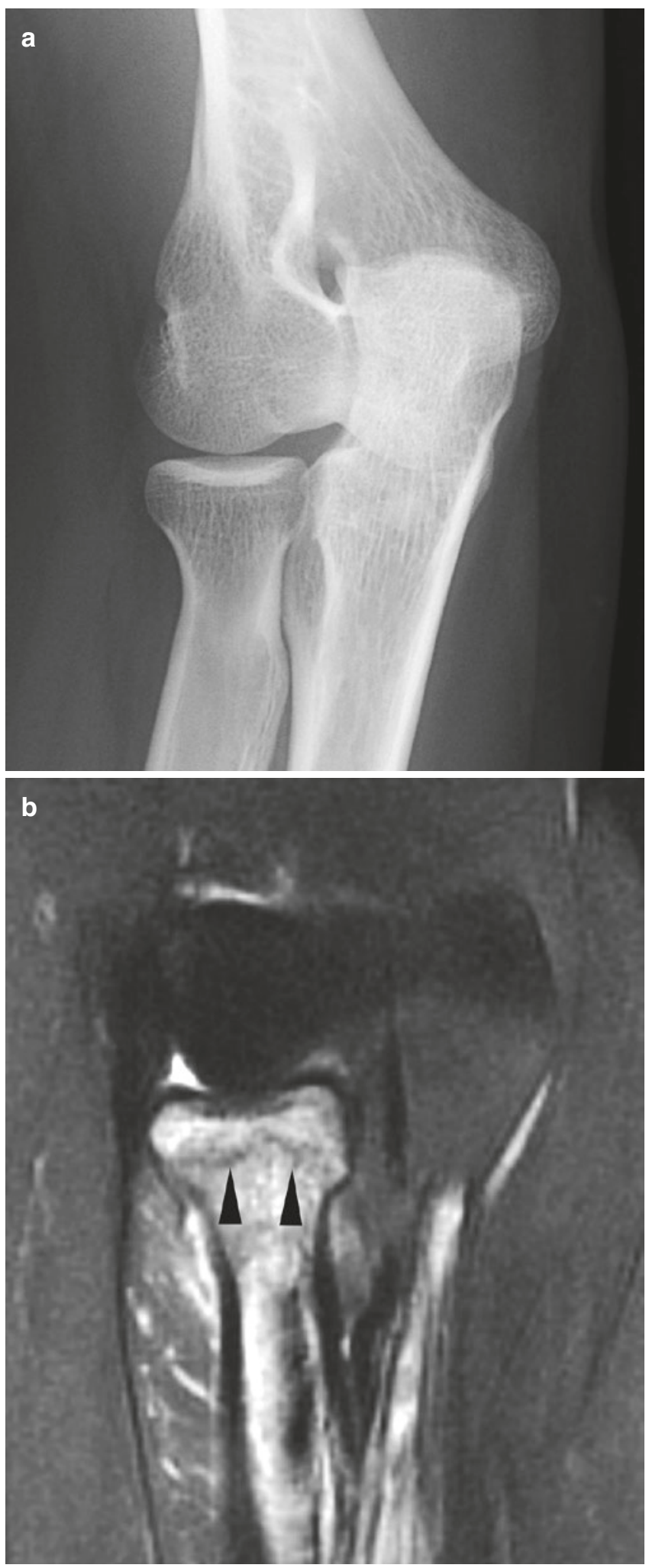

Fig. 3.3 Radiographically occult fracture. (a) Oblique radiograph of the elbow shows no evidence of fracture. (b) Coronal fat-saturated T2-weighted image reveals a non-displaced fracture of the radial head (arrowheads) with surrounding marrow edema. Used by permission of Springer Nature 


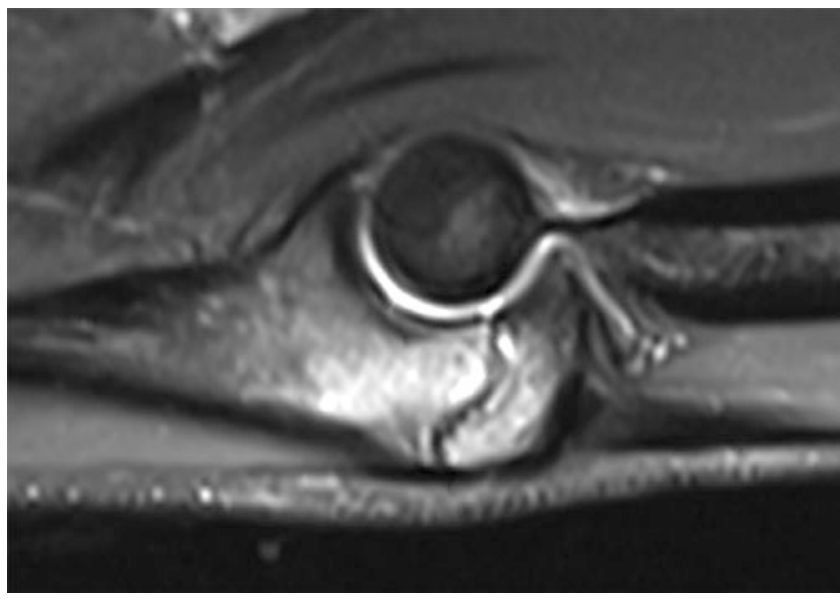

Fig. 3.4 Stress fracture. A 17-year-old male with 6-month history of posterior elbow pain exacerbated by weight lifting. Sagittal STIR image demonstrates a non-displaced fracture and prominent marrow edema in the olecranon. The olecranon physis was found to be fused in the opposite elbow on radiographs

tendon or forces along the posteromedial joint that occur in the valgus overload syndrome (see below). MR imaging findings are those of stress injuries anywhere in the body with edema-like abnormal signal intensity in the marrow indicating a stress reaction with or without a fracture line (Fig. 3.4).

\subsubsection{Osteochondral Lesions}

Osteochondral lesions of the elbow are thought to result from repetitive impaction or shear forces that damage the articular cartilage and underlying subchondral bone. Formerly known as osteochondritis dissecans (OCD), these injuries occur most often in adolescent throwing athletes or gymnasts.

The repetitive valgus forces that occur at the elbow during the throwing motion result in tensile forces across the medial joint that may produce laxity or tearing of the ulnar collateral ligament and/or the common flexor tendon. As these medial stabilizers fail, impaction and shear forces occur across the lateral aspect of the joint resulting in injury to the cartilage and subchondral bone of the capitellum and, less commonly, radial head [9]. These osteochondral lesions most commonly involve the anterolateral capitellum but have also been described in the trochlea in young athletes [10].

On MR images, osteochondral lesions demonstrate abnormal subchondral signal intensity as well as irregularity of the chondral surface, disruption or irregularity of the subchondral bone plate, and/or the presence of a subchondral fragment (Fig. 3.5). The primary role of imaging is to provide information regarding the stability of the osteochondral fragment. MRI, with its excellent soft tissue contrast, allows

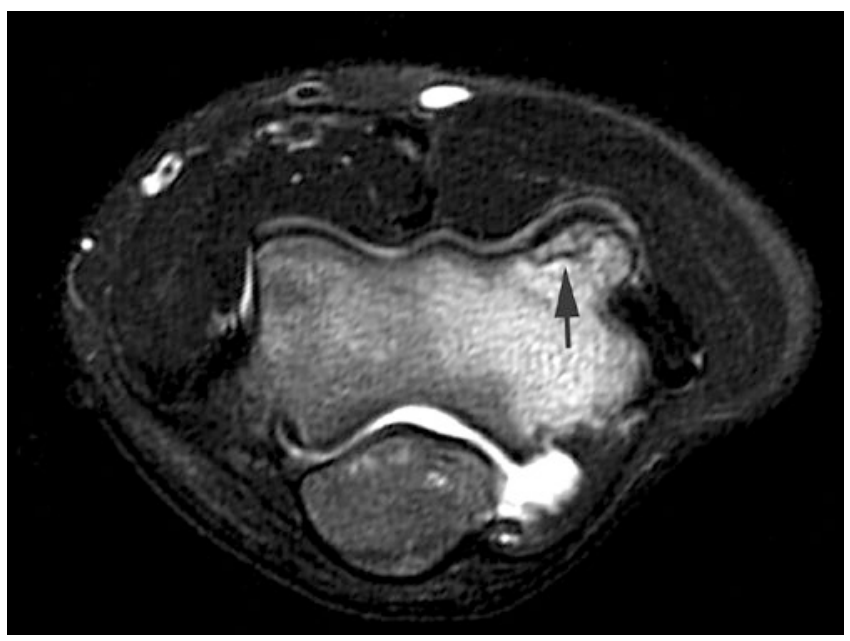

Fig. 3.5 Osteochondral lesion. A 14-year-old male baseball player with lateral elbow pain. Axial fat-saturated T2-wieghted image reveals extensive marrow edema and a subchondral fracture line in the anterior capitellum (arrow). The overlying cartilage appears to be intact, and there is no fluid undercutting the lesion to suggest this is an unstable fragment

direct visualization of the articular cartilage, as well as of the character of the interface of the osteochondral lesion with the native bone. The presence of joint fluid completely encircling the fragment generally indicates an unstable lesion [11]. Direct MR arthrography can be helpful in identifying an unstable fragment, and CT arthrography can also be used for this purpose.

\subsection{Ligaments}

\subsubsection{Ligaments: Normal Anatomy}

The ulnar collateral ligament (UCL) is made up of three bundles (Fig. 3.6). Its anterior bundle courses from the undersurface of the medial epicondyle to the sublime tubercle along the medial margin of the coronoid process. It is the most important stabilizer of the elbow, especially during throwing and other overhead motions. The posterior bundle of the UCL extends from the medial epicondyle in a fan-like distribution to the medial margin of the trochlear notch of the olecranon and forms the floor of the cubital tunnel. The small transverse bundle of the UCL runs from the olecranon to the coronoid process and does not play a significant role in stabilizing the elbow.

On the lateral side (Fig. 3.7), the radial collateral ligament (RCL) lies just deep to the common extensor tendon and courses from the lateral epicondyle to blend with the annular ligament along the volar aspect of the radiocarpal joint. The lateral ulnar collateral ligament (LUCL) also originates on 

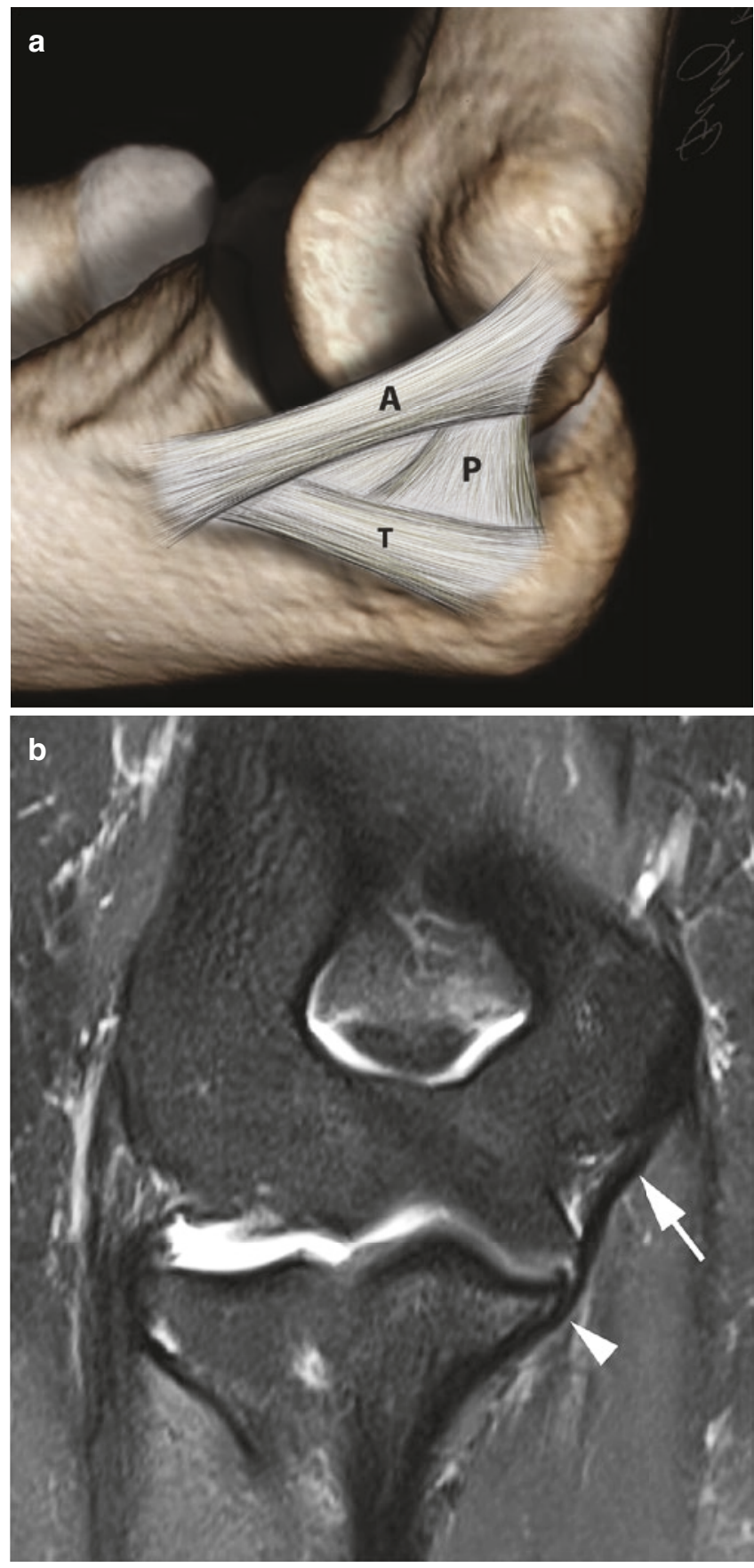

Fig. 3.6 Normal ulnar collateral ligament (UCL). (a) Diagram showing the anterior $(\mathrm{A})$, posterior $(\mathrm{P})$, and transverse $(\mathrm{T})$ bundles of the UCL. (b) The anterior bundle is well seen on a coronal fat-saturated T2-weighted image (arrow). Note its uninterrupted distal attachment at the sublime tubercle of the ulna (arrowhead). Illustration courtesy of Dr. Brady Huang, San Diego, CA

the lateral epicondyle and extends behind the radial head to attach to the supinator crest along the lateral aspect of the proximal ulna. It provides posterolateral stability for the radiocapitellar joint. The annular ligament runs from the ventral aspect of the radial notch of the ulna around the radial head to attach along with the LUCL along the supinator crest and stabilizes the proximal radioulnar joint [12]. Although these are described as individual structures, studies suggest that they function together as a continuous sheet, rather than as individual entities.

\subsubsection{Ligaments: Pathology}

\subsubsection{Valgus Instability}

The UCL and, in particular, its anterior band are the most important medial stabilizer of the elbow. The most common mechanisms of ulnar collateral ligament insufficiency are chronic attenuation, as seen in throwing or other overhead athletes, and acute post-traumatic disruption, usually after a fall on an outstretched arm.

During the throwing motion, high valgus stresses are placed on the elbow with resulting tensile forces along its medial aspect with the maximum stress on the ulnar collateral ligament occurring during the late cocking and acceleration phases [13]. Repetitive insults to the ligament produce microscopic tears that progress to significant attenuation or frank tearing within its substance. Complete, full-thickness tears are often well demonstrated on conventional MR imaging studies (Fig. 3.8), but partial tears can be subtle and are best seen with MR arthrography [14]. A partial-thickness undersurface tear will often result in a "T-sign" which is best demonstrated using MR arthrography (see Fig. 3.7).

In the skeletally immature patient, the apophysis is the weakest link in the muscle-tendon-bone complex. Chronic valgus stress may result in a condition known as "Little Leaguer's Elbow" in which repetitive stresses across the medial epicondylar apophysis result in its apparent widening on radiographs (also known as "epiphysiolysis"), displacement or fragmentation [15]. MR imaging demonstrates extension of physeal cartilage into the metaphysis and/or marrow edema within the apophysis and also allows for assessment of the UCL, which is usually normal in these patients (Fig. 3.9).

\subsubsection{Valgus Overload Syndrome}

Throwing athletes and others involved in overhead sports often develop a constellation of injuries known as the valgus overload syndrome [16]. This is related to the strong valgus forces generated at the elbow which may lead to tensile failure of the UCL, an osteochondral lesion in the capitellum and/or subchondral edema, cartilage loss, and osteophyte formation along the posteromedial elbow joint secondary to increased shear forces in that region (Fig. 3.10). A careful inspection of these three areas should be carried out when viewing an MR imaging study in an overhead athlete presenting with elbow pain. 

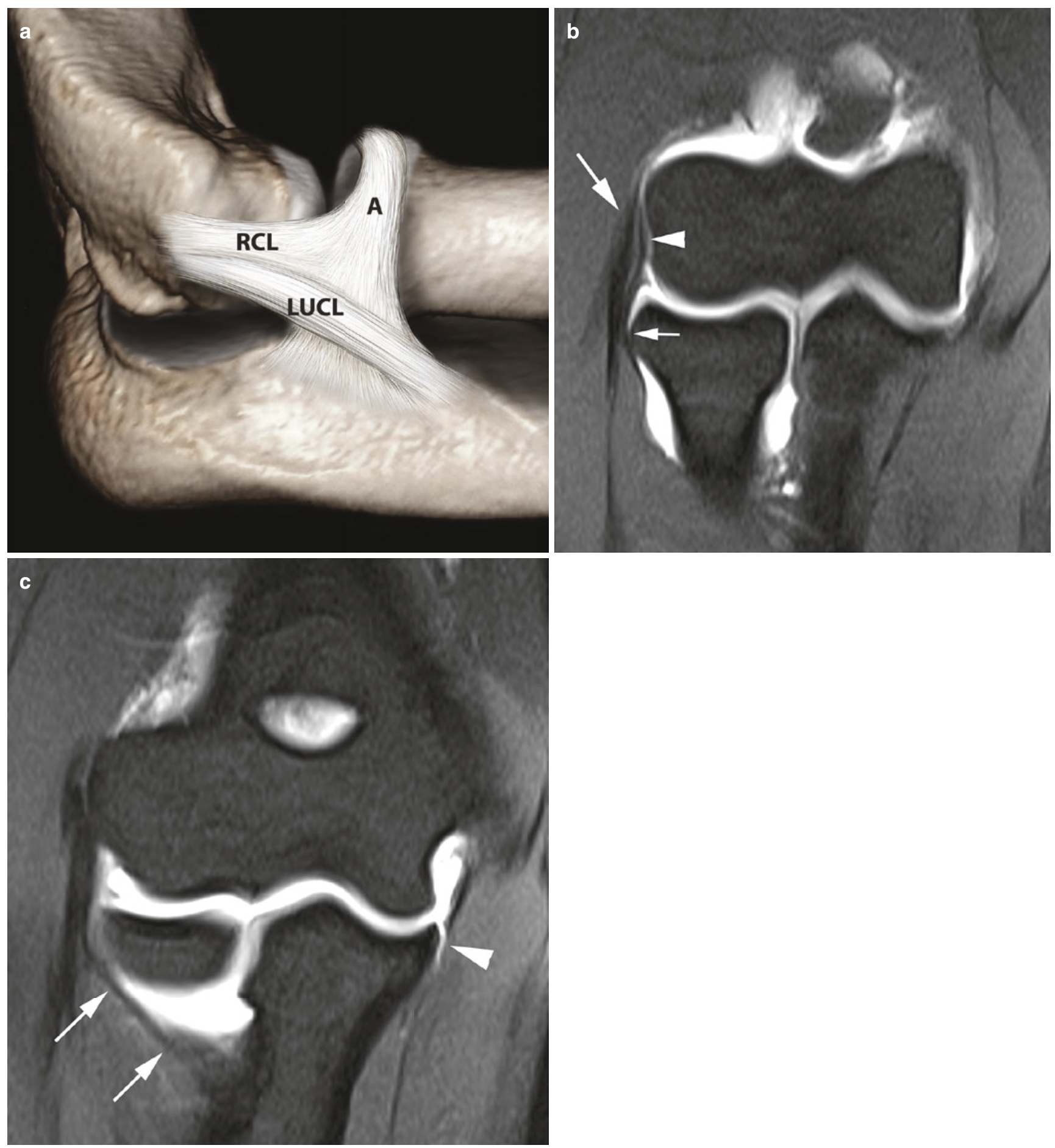

Fig. 3.7 Normal lateral ligaments. (a) Diagram showing the lateral collateral ligament complex including the radial collateral ligament (RCL), lateral ulnar collateral ligament (LUCL), and annular ligament (A). (b) Coronal fat-saturated T1-weighted image (MR arthrogram) shows the RCL (arrowhead) just deep to the common extensor tendon (arrow) and merging distally with the annular ligament (small arrow). (c) A more posterior image demonstrates the LUCL (arrows) as it courses behind the radial head to insert on the supinator crest of the ulna. Note also a partial tear of the distal ulnar collateral ligament forming a "T sign" (arrowhead). Illustration courtesy of Dr. Brady Huang, San Diego, CA 


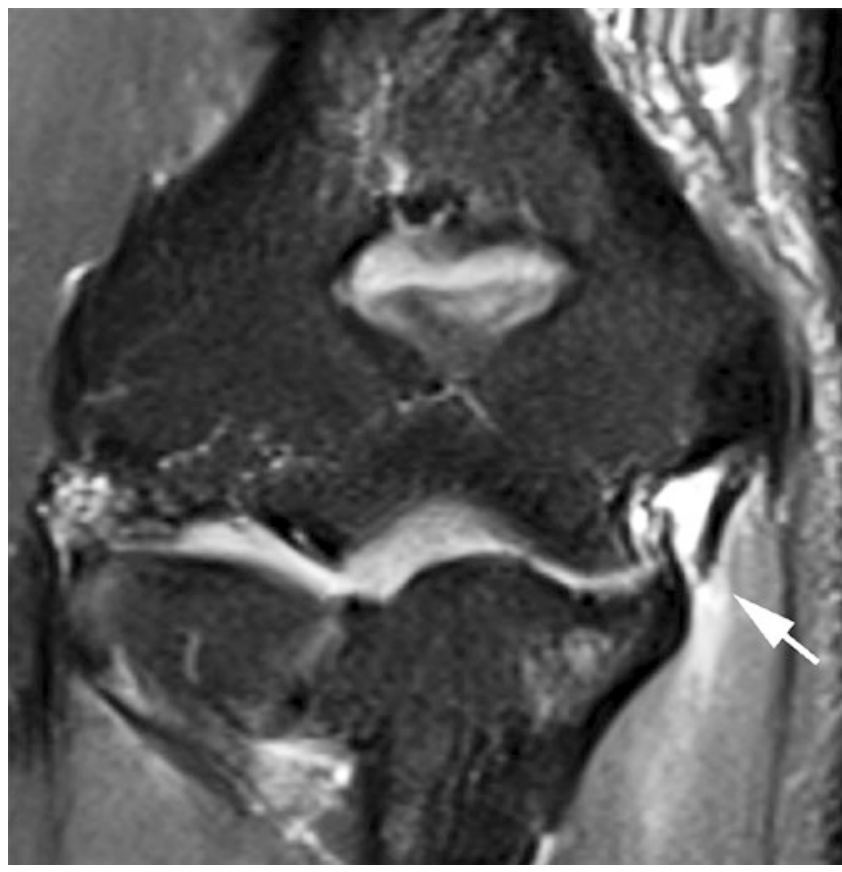

Fig. 3.8 Ulnar collateral ligament tear. An 18-year-old college baseball pitcher who felt a "pop" in his elbow while throwing and presented with medial elbow pain. Coronal fat-saturated T2-weighted image reveals a full-thickness tear of the anterior bundle of the UCL at its ulnar attachment (arrow) and edema in the adjacent flexor musculature

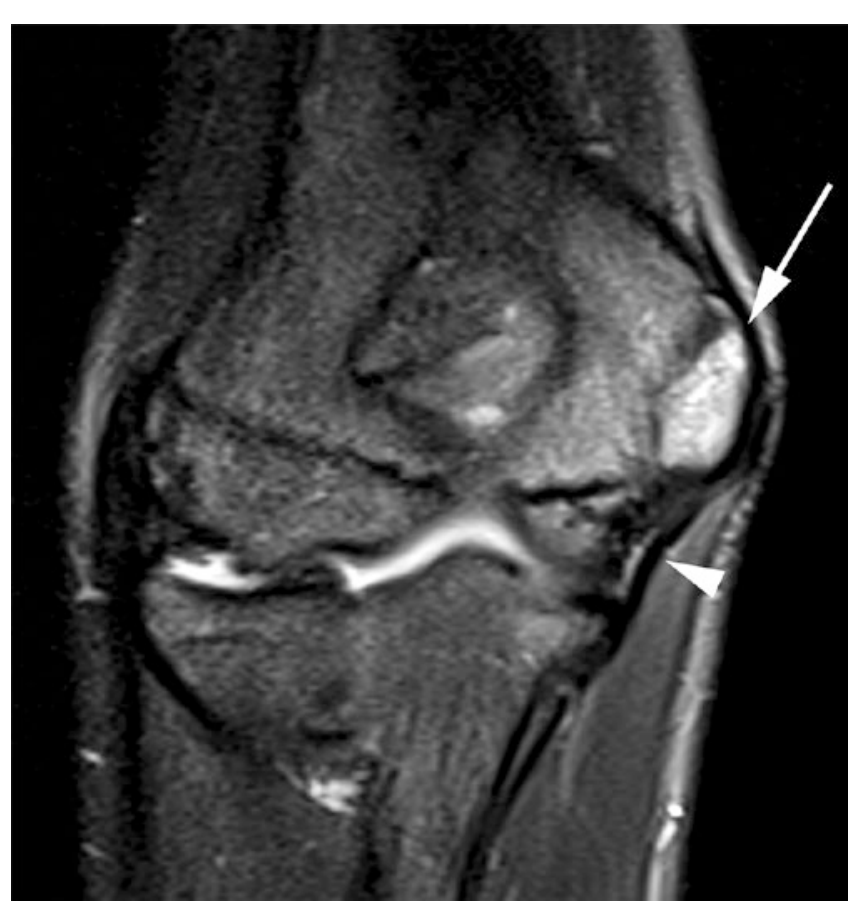

Fig. 3.9 Medial apophysitis ("Little Leaguer's Elbow"). Coronal fatsaturated T2-weighted image (MR arthrogram) displays marrow edema within the medial epicondylar apophysis (arrow) in a 14-year-old baseball player. Note also the normal UCL (arrowhead)

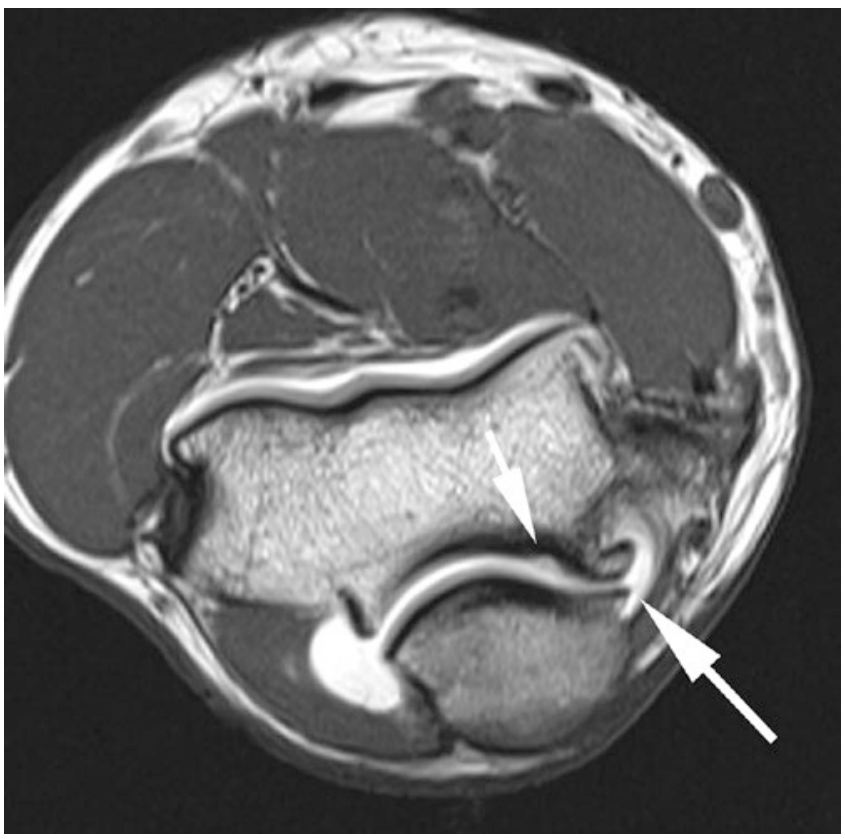

Fig. 3.10 Valgus overload syndrome: posteromedial joint. Axial T1-weighted image (MR arthrogram) demonstrates prominent osteophytes along the posteromedial joint (large arrow) as well as a small focus of cartilage loss along the posterior trochlea (small arrow). Used by permission of Springer Nature

\subsubsection{Posterolateral Rotatory Instability and Elbow Dislocation (Fig. 3.11)}

Posterolateral rotatory instability (PLRI) is the most common pattern of recurrent elbow instability and is often the sequela of a prior elbow dislocation. With an elbow dislocation, soft tissue injuries classically have been thought to proceed from lateral to medial along a spectrum of severity consisting of three stages: stage 1 , posterolateral subluxation of the ulna on the humerus resulting in injury to the lateral ulnar collateral ligament; stage 2, incomplete dislocation with perching of the coronoid under the trochlea and tearing of the radial collateral ligament and joint capsule in addition to the lateral ulnar collateral ligament; and stage 3, complete dislocation with the coronoid located behind the humerus. Severe injuries may also result in additional tearing of the ulnar collateral ligament [17].

In addition to the soft tissue injuries, fractures involving the coronoid process and radial head commonly occur with an elbow dislocation, and the constellation of findings is referred to as the "terrible triad" of the elbow [18]. A fracture of the coronoid process is pathognomonic of an episode of elbow subluxation or dislocation, and the larger the coronoid fracture fragment, the more pronounced the degree of posterolateral instability. 

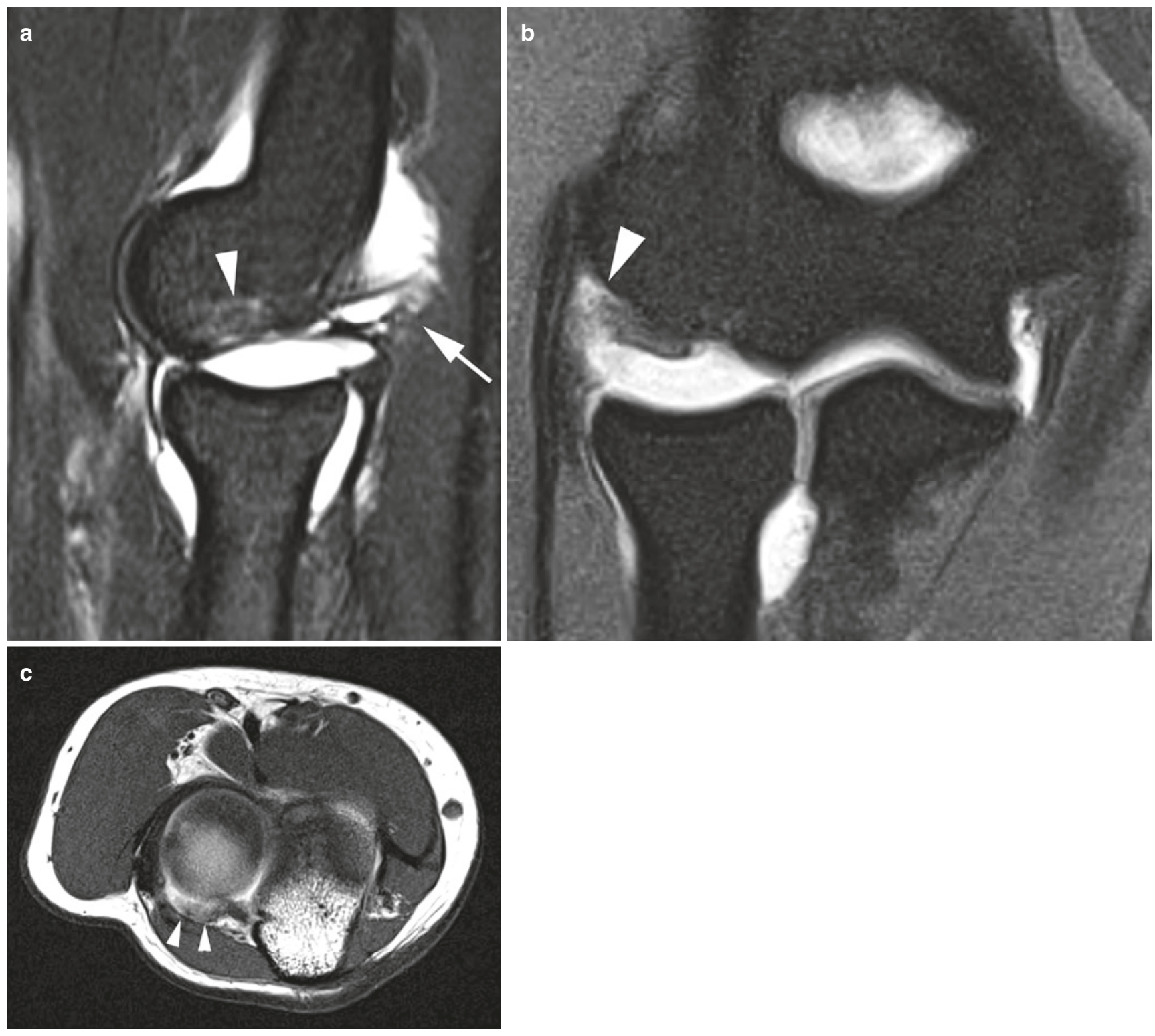

Fig. 3.11 Elbow dislocation. (a) Sagittal STIR image (MR arthrogram) shows posterior subluxation of the radial head and posterior ligamentous/capsular tearing (arrow) as well a subtle bone marrow contusion in the posterior capitellum (arrowhead). (b) Coronal fat-

saturated T1-weighted image displays high-grade tearing of the RCL from its humeral attachment and an axial T1-weighted image (c) reveals tearing of the LUCL distally (arrowheads)

\section{Key Point}

- Stabilization of the elbow occurs primarily through static stabilizers of the ligament complexes and the intrinsic stability conferred by its osseous anatomy. Acute and chronic repetitive traumas are commonly encountered and have MR findings that vary with regard to skeletally maturity. Structural failure can be predicted with known mechanism of injury.

\subsection{Tendons and Muscles}

\subsubsection{Tendons and Muscles: Normal Anatomy (Fig. 3.12)}

The biceps tendon courses across the elbow anteriorly to insert on the radial tuberosity and is best seen in the axial plane. The bicipital aponeurosis (lacertus fibrosus) is a fascial extension from the short head of the biceps that extends 

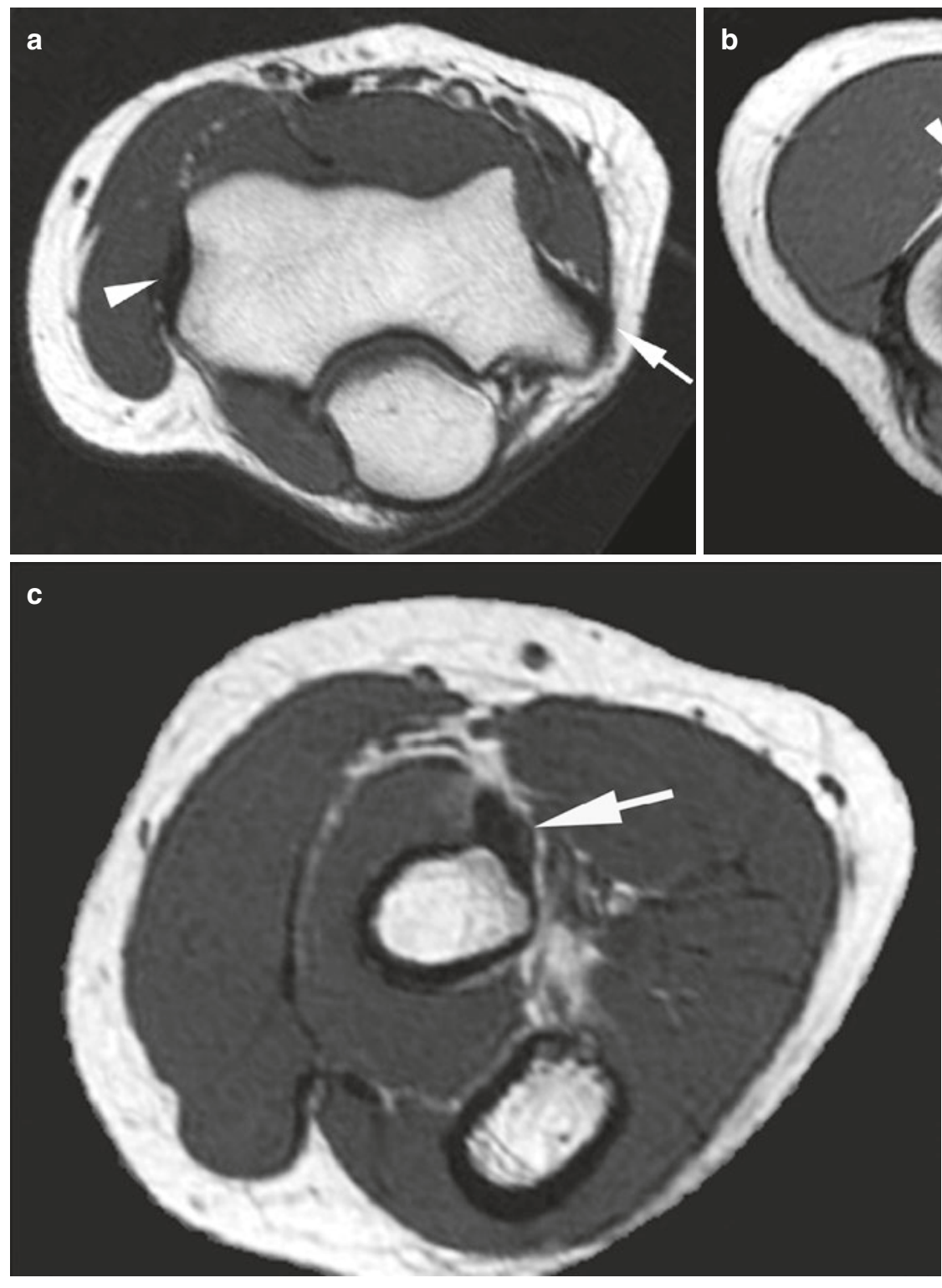

Fig. 3.12 Normal tendons. Axial T1-weighted images display (a) the common flexor/pronator (arrow) and common extensor (arrowhead) tendons, (b) the biceps tendon (large arrow), lacertus fibrosis/bicipital aponeurosis (black arrowhead), brachialis tendon (small arrow), branches of the radial nerve within the radial tunnel (white arrowheads),

over the biceps tendon and provides some tendon stability in this region. Immediately deep to the biceps lies the brachialis muscle and its very short tendon that attaches to the ventral aspect of the coronoid process of the ulna.

Posteriorly, the triceps tendon attaches along the dorsal aspect of the olecranon, distal to its tip, and can be divided into a thicker central tendon and a thinner lateral expansion. The small anconeus muscle lies along the posterolateral aspect of the elbow, arising from the posterior aspect of the lateral epicondyle and inserting along the proximal ulna. The
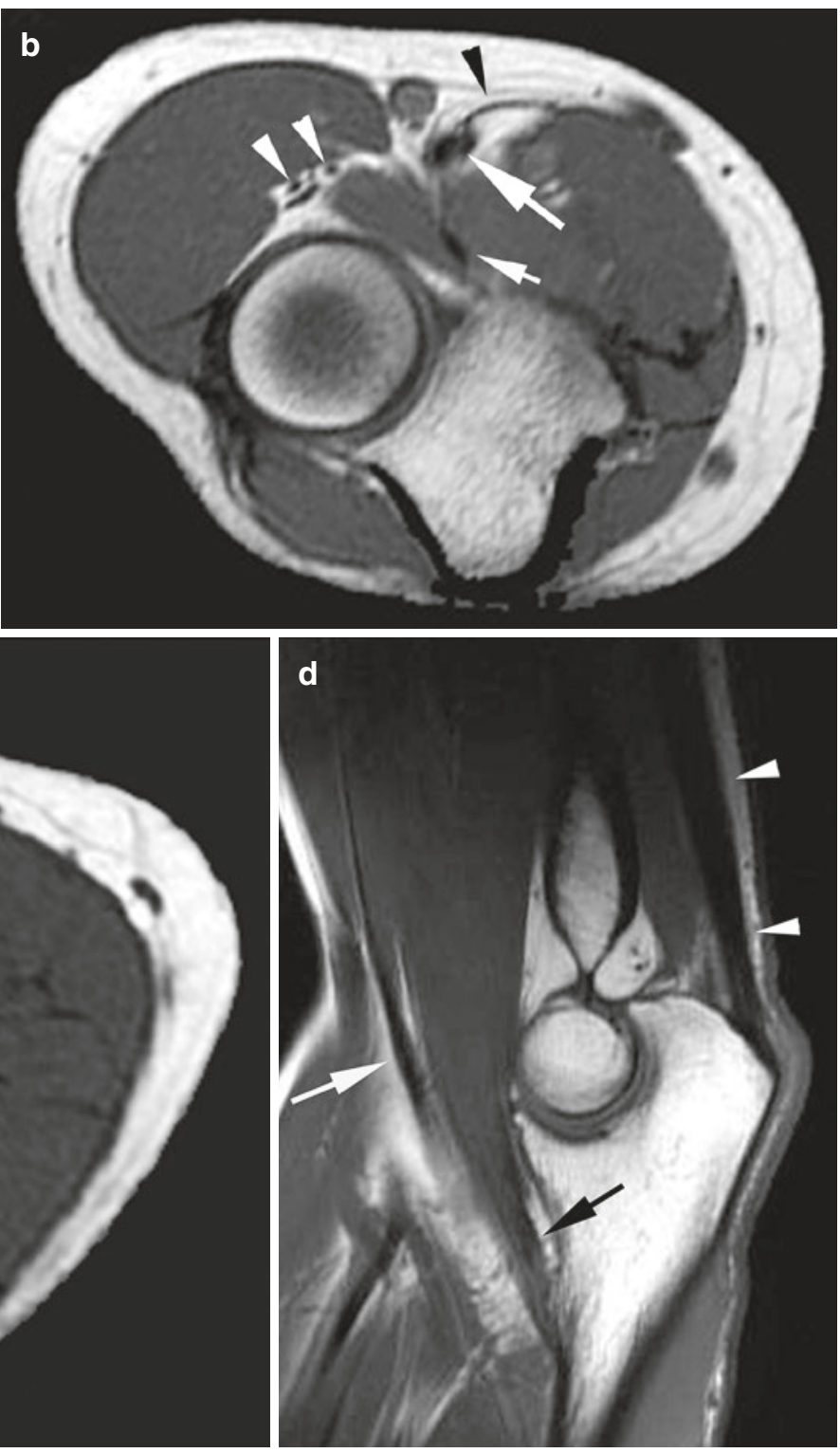

and (c) the distal biceps tendon at its insertion on the radial tuberosity (arrow). (d) Sagittal T1-weighted image again demonstrates the biceps (white arrow) and brachialis (black arrow) tendons as well as the triceps tendon (arrowheads). Used by permission of Springer Nature

anconeus epitrochlearis is an anomalous muscle, seen in approximately $10 \%$ of individuals, that lies along the medial aspect of the olecranon. While this is usually an asymptomatic normal variant, it may produce symptoms of ulnar neuropathy due to compression of the nerve within the adjacent cubital tunnel (see Fig. 3.17).

The flexor/pronator muscles of the forearm originate via a common tendon from the medial epicondyle, while the common extensor/supinator tendon originates on the lateral epicondyle. 


\subsubsection{Tendon and Muscles: Pathology}

The classification of tendon injuries about the elbow can be organized by location, acuity, and degree of injury. Tendon injury related to a single isolated event is uncommon, although exceptions to this rule do occur. More commonly, tendon pathology in the elbow relates to chronic repetitive micro-trauma. MR imaging and ultrasound are particularly well suited to diagnose tendon pathology.

As elsewhere in the body, the tendons about the elbow should be smooth, linear structures of low signal intensity on MRI [19]. Abnormal morphology (attenuation or thickening) can be seen in tendinosis (also termed tendinopathy) or tear. If signal intensity becomes increased within the substance of a tendon on fluid-sensitive sequences, a tear is present. Tears can be further characterized as partial or complete.

\subsubsection{Epicondylitis and Overuse Syndromes}

Chronic stresses applied to the common flexor and extensor tendons result in medial and lateral "epicondylitis," though this is a misnomer since the underlying pathology relates to chronic degeneration and partial tendon tearing rather than an acute inflammatory reaction. The injury is believed to result from repetitive tensile overload of the tendon that produces microscopic tears that do not heal appropriately.

\subsubsection{Lateral Epicondylitis}

Lateral epicondylitis is associated with excessive, repetitive use of the wrist extensors and is the most common athletic injury in the elbow. It has been termed "tennis elbow," but this is somewhat misleading since the vast majority of cases of lateral epicondylitis occur in non-tennis players. The pathology most commonly affects the extensor carpi radialis brevis at the origin of the common extensor tendon.

The imaging findings of epicondylitis may include tendon thickening or attenuation with intrasubstance intermediate signal intensity (tendinosis) or partial- or full-thickness tears in which case fluid signal intensity will be seen within the tendon (Fig. 3.13). Close scrutiny of the underlying lateral ligaments is necessary to exclude concomitant pathology, especially in the lateral ulnar collateral ligament [20]. Underlying ligament involvement predisposes to a poorer

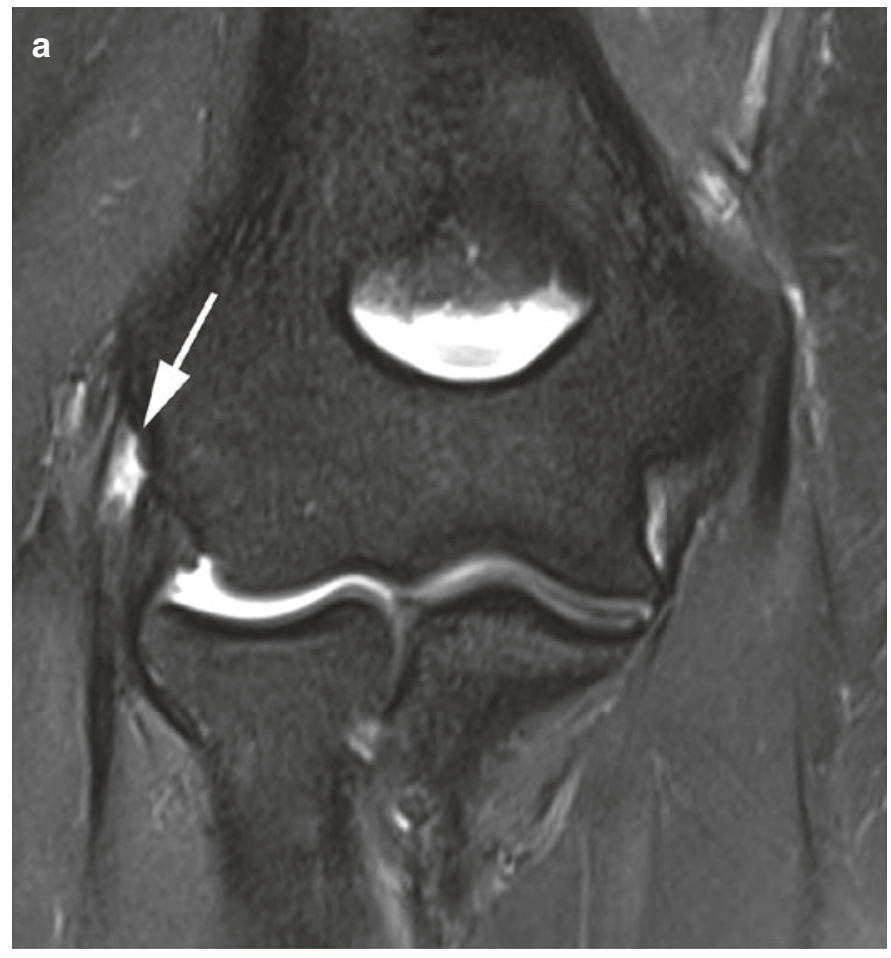

Fig. 3.13 Lateral epicondylitis. (a) Coronal fat-saturated T2-weighted image displays partial tearing of the proximal common extensor tendon at its humeral origin (arrow). (b) An adjacent image reveals partial tear-

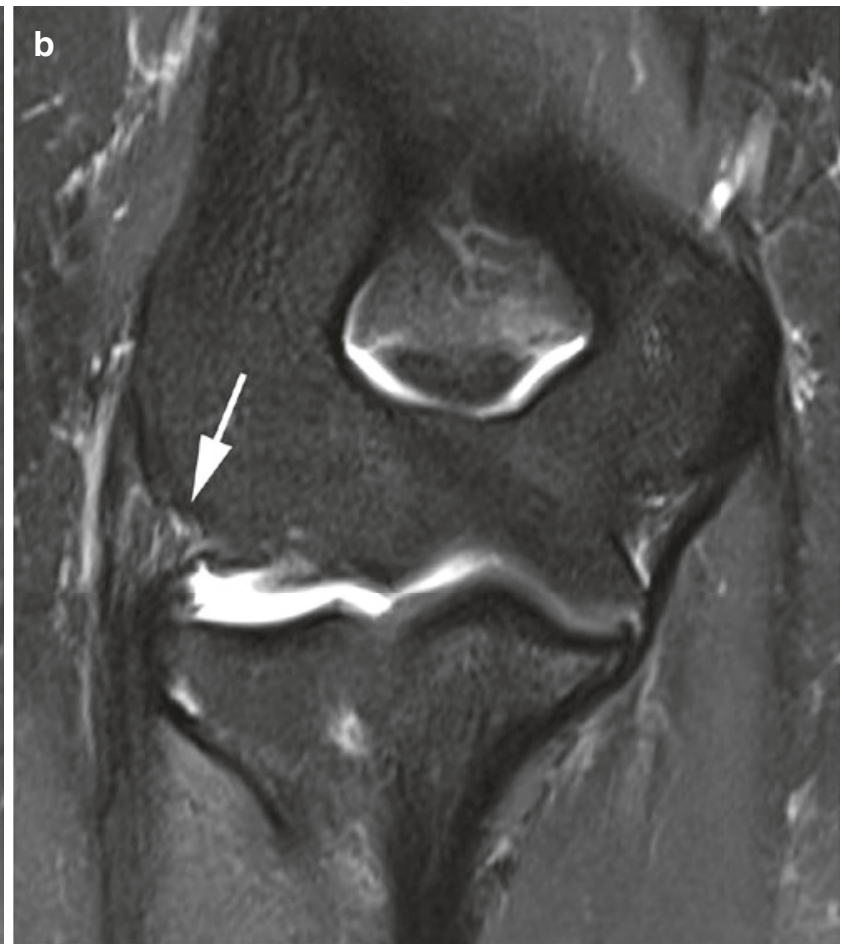

ing of the underlying humeral attachment of the radial collateral and lateral ulnar collateral ligaments (arrow) 
response to conservative therapy and is an important finding for preoperative planning if surgical debridement of the common extensor tendon is contemplated.

\subsubsection{Medial Epicondylitis}

Medial "epicondylitis" involves a similar degenerative process affecting the common flexor tendon and results from repetitive valgus strain. It is associated primarily with golfing, pitching, and tennis. The pronator teres and flexor carpi radialis tendons are involved most frequently, resulting in pain and tenderness to palpation over the anterior aspect of the medial epicondyle and at the origin of the common flexor tendon. The imaging findings in this process are exactly those seen with lateral epicondylitis. As on the lateral side, when assessing the tendon, it is important to closely scrutinize the underlying collateral ligament complex to ensure its integrity.

\subsubsection{Biceps Tendon}

Rupture of the tendon of the biceps brachii muscle at the elbow constitutes less than $5 \%$ of all biceps tendon injuries with the most common injury in this region being complete avulsion of the tendon from the radial tuberosity [21]. The typical mechanism of injury relates to forced hyperextension applied to a flexed and supinated forearm, and the patient often describes sensing a "pop" and sharp pain in the antecubital fossa. Accurate clinical diagnosis is difficult in a partial tendon tear or in the case of a complete tear without retraction which can occur with an intact bicipital aponeurosis, which tethers the ruptured tendon to the pronator flexor muscle group.

Imaging of distal biceps tendon pathology becomes important in patients who do not present with the classic history or physical findings. It is critical that axial MR images extend distal to the radial tuberosity for accurate evaluation. MRI diagnosis of tendon pathology, as previously mentioned, is largely dependent on morphology, signal intensity, and the identification of areas of tendon discontinuity. The degree of partial tearing should be described and, in the case of a complete tear, the degree of tendon retraction and status of the bicipital aponeurosis. A useful indirect sign of biceps tendon pathology is the presence of radiobicipital bursitis (see below) (Fig. 3.14).

\subsubsection{Triceps Tendon}

Rupture of the triceps tendon is quite rare [22]. This most often results from an acute injury and is most common in weight lifters and other athletes. Partial tears are more common than complete tears, and both usually occur at its olecranon insertion. Associated findings may include olecranon bursitis, subluxation of the ulnar nerve, or fracture of the radial head (Fig. 3.15). The MRI features of a tear are similar to those associated with any other tendon.
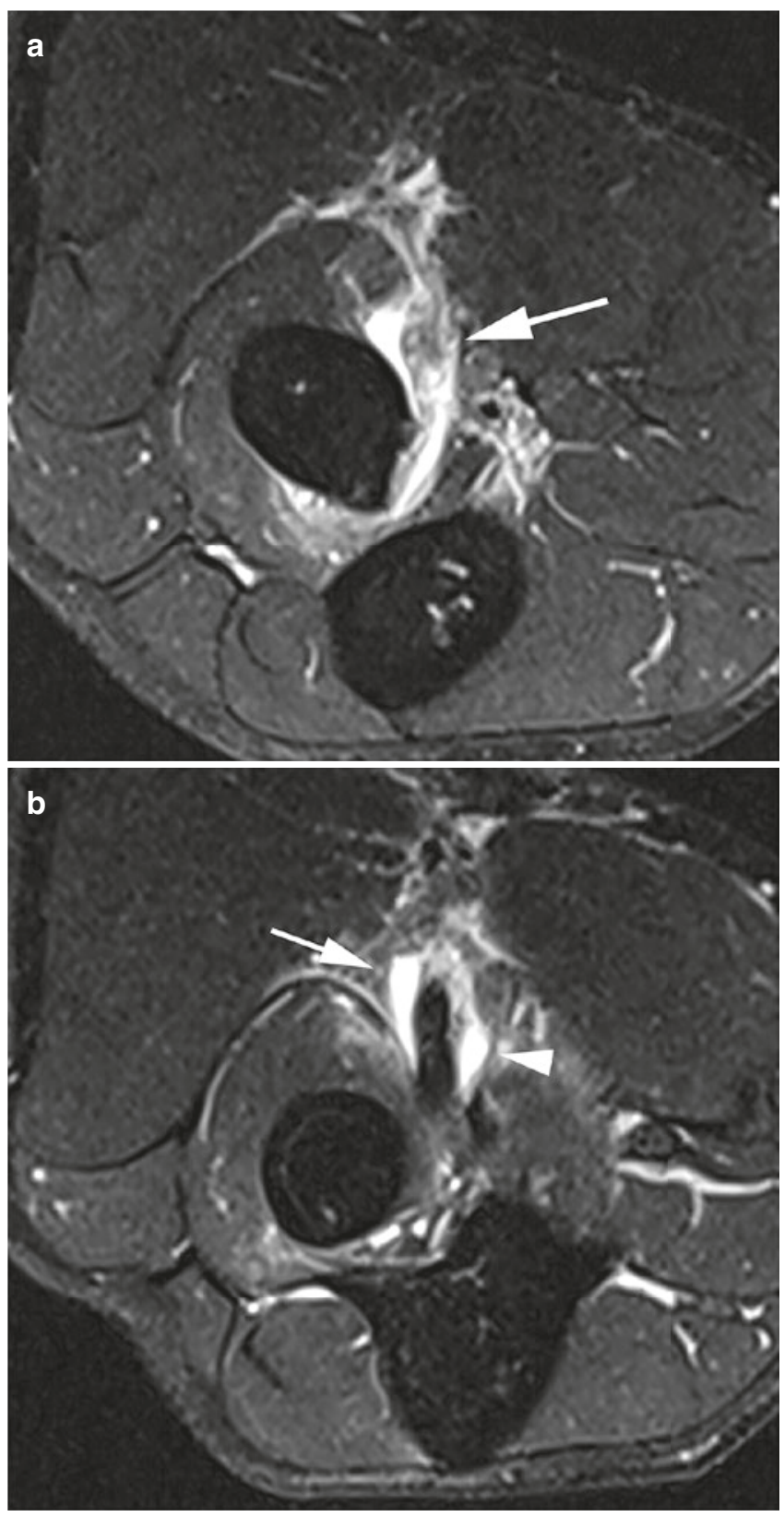

Fig. 3.14 Biceps tendon tear. (a) Axial fat-saturated T2-weighted image shows near complete tearing of the biceps tendon from the radial tuberosity (arrow). A more proximal image (b) demonstrates some associated distention of the radiobicipital bursa (arrow) and interosseous bursa (arrowhead)

\subsection{Nerves}

\subsubsection{Nerves: Normal Anatomy (Fig. 3.16)}

The ulnar nerve courses along the periphery of the medial head of the triceps muscle in the distal portion of the upper arm and into the cubital tunnel along the posteromedial elbow where it lies superficial to the posterior bundle of the UCL and deep to the cubital tunnel retinaculum. 


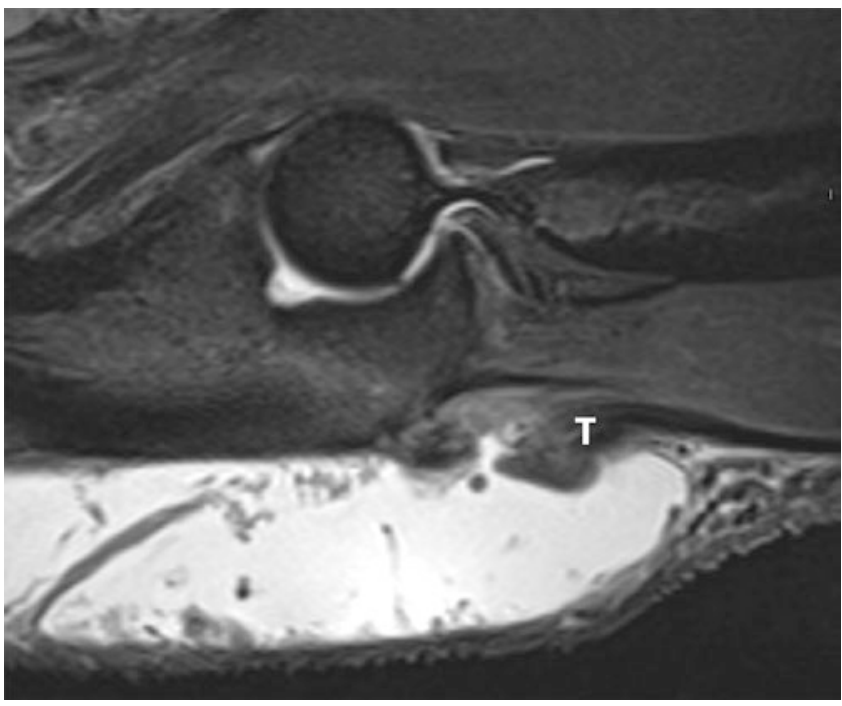

Fig. 3.15 Triceps tendon tear. Sagittal STIR image demonstrates partial tearing of the distal triceps tendon (T) and extensive fluid and synovial hypertrophy within the olecranon bursa

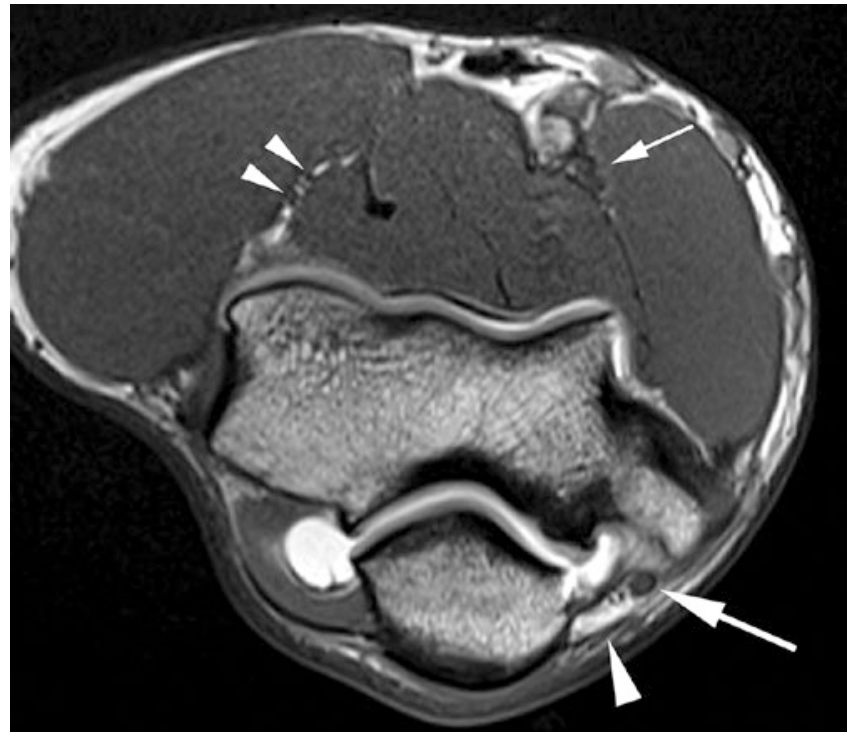

Fig. 3.16 Normal nerves. Axial T1-weighted image (MR arthrogram) demonstrates the ulnar nerve within the cubital tunnel (large arrow) and overlying cubital tunnel retinaculum (large arrowhead), the superficial and deep branches of the radial nerve within the radial tunnel (arrowheads), and the median nerve (small arrow). Used by permission of Springer Nature

After spiraling around the midhumeral shaft, the radial nerve courses through the radial tunnel, a space along the volar-lateral aspect of the elbow between the brachioradialis and brachialis muscles, where it divides into its deep and superficial branches. The deep branch then passes under the arcade of Froshe, pierces the supinator muscle, and continues as the posterior interosseous nerve.
The median nerve travels along the medial upper arm with the brachial vessels and then courses between the two heads of the pronator teres muscle and gives off the anterior interosseous nerve more distally.

\subsubsection{Nerves: Pathology}

Pathology involving the nerves of the upper extremity may result from nerve compression/entrapment or a "nonentrapment" etiology such as infection, polyneuropathy, acute trauma, or iatrogenic injury during arthroscopy [23]. Most types of nerve pathology will result in changes within the muscles they innervate: high signal intensity "edema" on $\mathrm{T} 2 \mathrm{~W}$ images with an acute process and high signal intensity on T1-weighted images, indicating fatty atrophy, with more chronic processes. In many cases, these muscle findings are more conspicuous on MR images than an abnormality of the nerve itself.

\subsubsection{Ulnar Nerve}

Ulnar nerve entrapment most commonly occurs in the cubital tunnel and may arise from a number of etiologies including a medial trochlear osteophyte, an anomalous anconeus epitrochlearis muscle, or an adjacent soft tissue mass (Fig. 3.17).

On MR imaging, ulnar neuritis should be considered when the nerve is enlarged, contains fascicles of varying size, and demonstrates increased signal intensity on $\mathrm{T} 2 \mathrm{~W}$ images, although this latter sign can be seen in asymptomatic patients and therefore must be correlated with other imaging and clinical information [24].

If the ulnar nerve is not seen within the cubital tunnel, it has likely been previously surgically transposed to the volar aspect of the elbow either deep or superficial to the proximal flexor muscles. To locate the nerve after transposition, it is easiest to identify it on T1-weighted images in the medial musculature of the proximal forearm where it is surrounded by bright fat and then trace its course proximally from there.

\subsubsection{Median Nerve}

Compression of the median nerve may occur at several sites. In patients with a supracondylar process emanating from the anterior cortex of the distal humerus, the nerve may become compressed by an associated ligament of Struthers (supracondylar process syndrome). The nerve may also become entrapped as it passes between the two heads of the pronator teres muscle or under the fibrous arch of the flexor digitorum profundus (pronator syndrome). More distally, the anterior interosseous nerve may be injured or compressed by an adjacent mass (anterior interosseous nerve syndrome). 

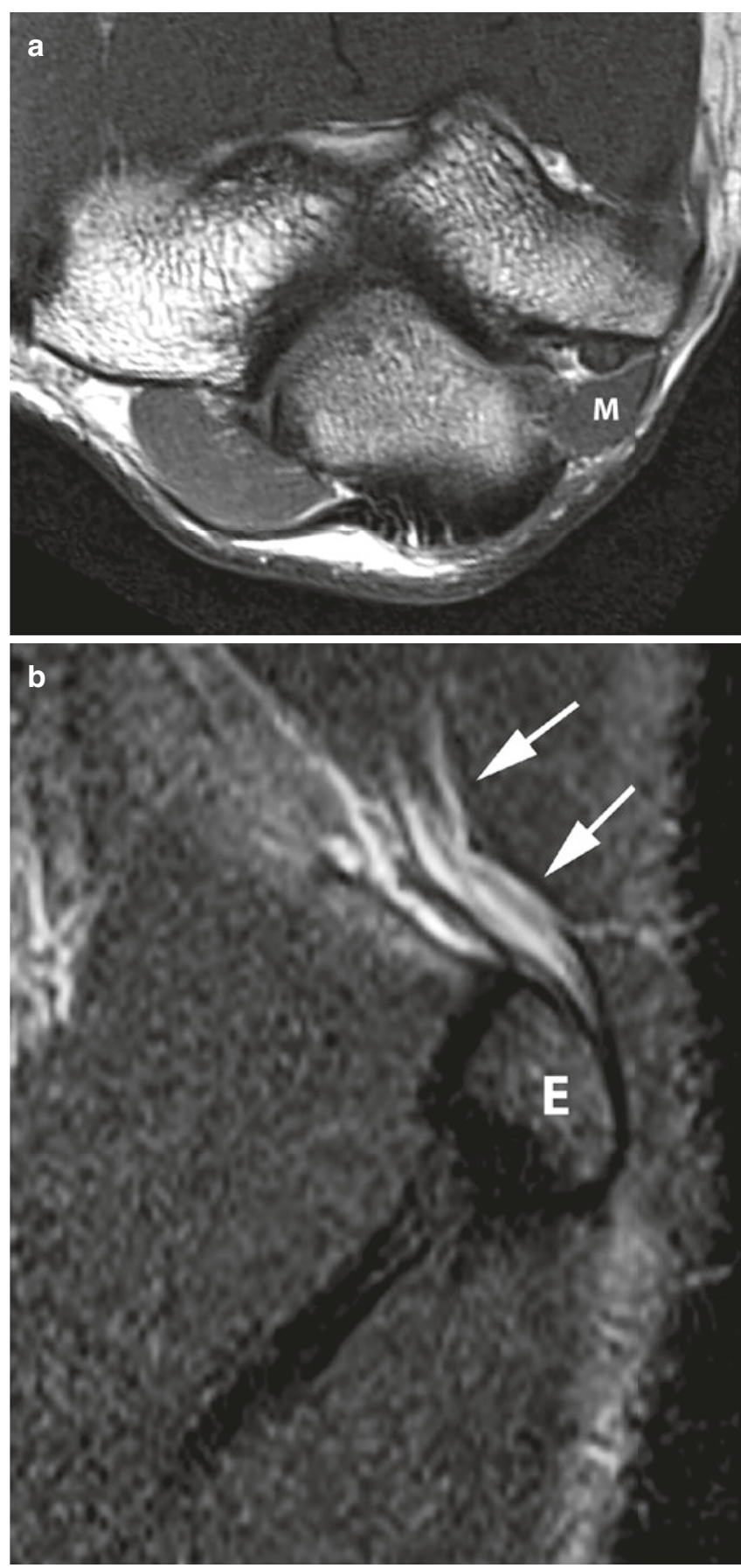

Fig. 3.17 Ulnar neuritis. A 31-year-old male with elbow pain and symptoms of ulnar neuropathy. (a) Axial T1-weighted image reveals an anconeus epitrochlearis muscle (M) superficial to the ulnar nerve within the cubital tunnel. (b) Sagittal STIR image shows an increased signal intensity within a markedly enlarged ulnar nerve just proximal to the cubital tunnel (arrows) $(\mathrm{E}=$ medial epicondyle)

\subsubsection{Radial Nerve}

The radial nerve may be injured from direct trauma or may be compressed at various sites, the most common being where it penetrates the supinator muscle at the arcade of Frohse, a fibrous band found at that level in $30-50 \%$ of

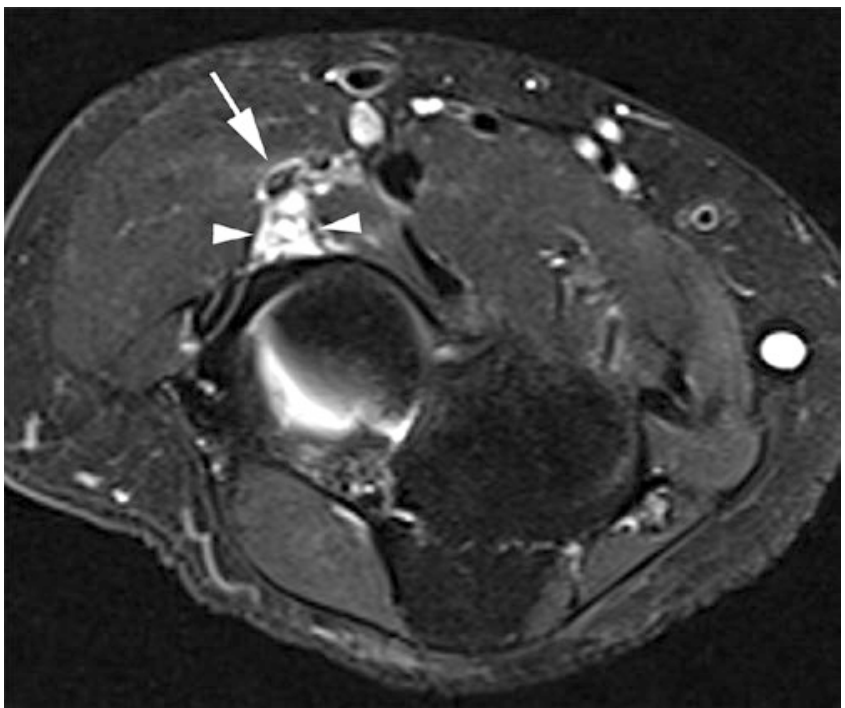

Fig.3.18 Radial nerve impingement. A 46-year-old female presenting with symptoms of posterior interosseous syndrome. Axial fat-saturated T2-weighted image shows a multilocular ganglion (arrowheads) displacing the deep branch of the radial nerve at that level (arrow)

patients. The nerve may also be compressed by an adjacent space-occupying mass (Fig. 3.18).

\subsection{Synovial Processes}

As a synovial joint, the elbow has a synovial membrane and joint capsule, separated by fat pads in some locations. Lubricating bursae, lined by synovium, are located superficial to the olecranon and surrounding the distal biceps tendon. The synovial anatomy is further complicated by the synovial plicae, folds of synovial tissue that represent embryonic remnants. Synovial inflammation can result from infectious, inflammatory, or post-traumatic conditions. Its appearance on MR imaging is nonspecific and includes T2-hyperintense joint fluid and synovial hypertrophy with enhancement if intravenous gadolinium is administered.

\subsubsection{Bursae}

The large olecranon bursa lies in the subcutaneous tissues overlying the dorsal aspect of the proximal ulna [25]. Two other bursae lie adjacent to the distal biceps tendon: the radiobicipital and interosseous bursae [26]. It is important to note that there is no tendon sheath at the level of the distal biceps tendon, so peritendinous fluid in this region indicates distention of one or both of these bursae.

Bursal inflammation may result from a number of etiologies which will be discussed below. MR imaging findings include bursal distention with high signal intensity fluid or, 


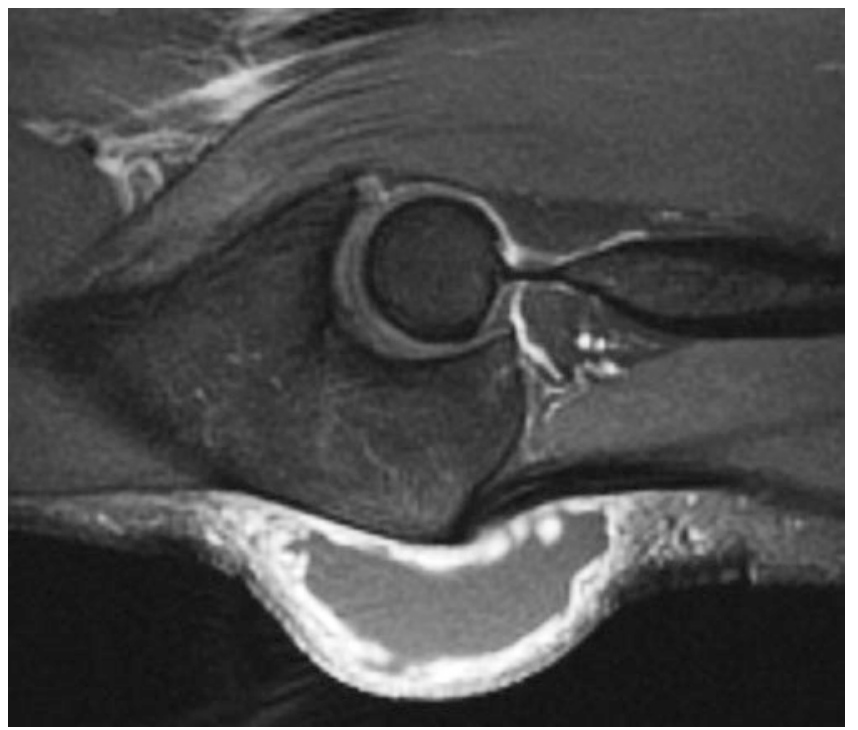

Fig. 3.19 Olecranon bursitis. Sagittal fat-saturated T1-weighted image demonstrates marked synovial enhancement in a distended olecranon bursa. Used by permission of Springer Nature

in the case of infection or hemorrhage, more heterogeneous material on $\mathrm{T} 2 \mathrm{~W}$ images and pronounced synovial enhancement on T1-weighted images after the intravenous administration of gadolinium contrast (Fig. 3.19).

\subsubsection{Plicae}

Synovial plicae represent embryologic remnants that are commonly found within asymptomatic elbows. The most common of these synovial folds is the posterolateral plica, which is present in $86 \%$ of cadavers and up to $98 \%$ of asymptomatic patients on MR imaging; it has been implicated as a potential cause of lateral elbow pain known as the synovial fold syndrome [27]. This is thought to result from dynamic entrapment of the plica between the radial head and capitellum and often produces snapping, popping, or even locking of the elbow. The MR imaging findings of a plica measuring greater than $3 \mathrm{~mm}$ in thickness or covering more than one third of the articular surface of the radial head should raise suspicion for the diagnosis (Fig. 3.20).

\subsubsection{Synovial Inflammation}

Synovial inflammation can result from infectious, inflammatory, or post-traumatic conditions. The appearance on MR imaging is nonspecific and includes T2-hyperintense joint effusion, synovial hypertrophy, and synovial enhancement if intravenous gadolinium is administered [28] (Fig. 3.21).
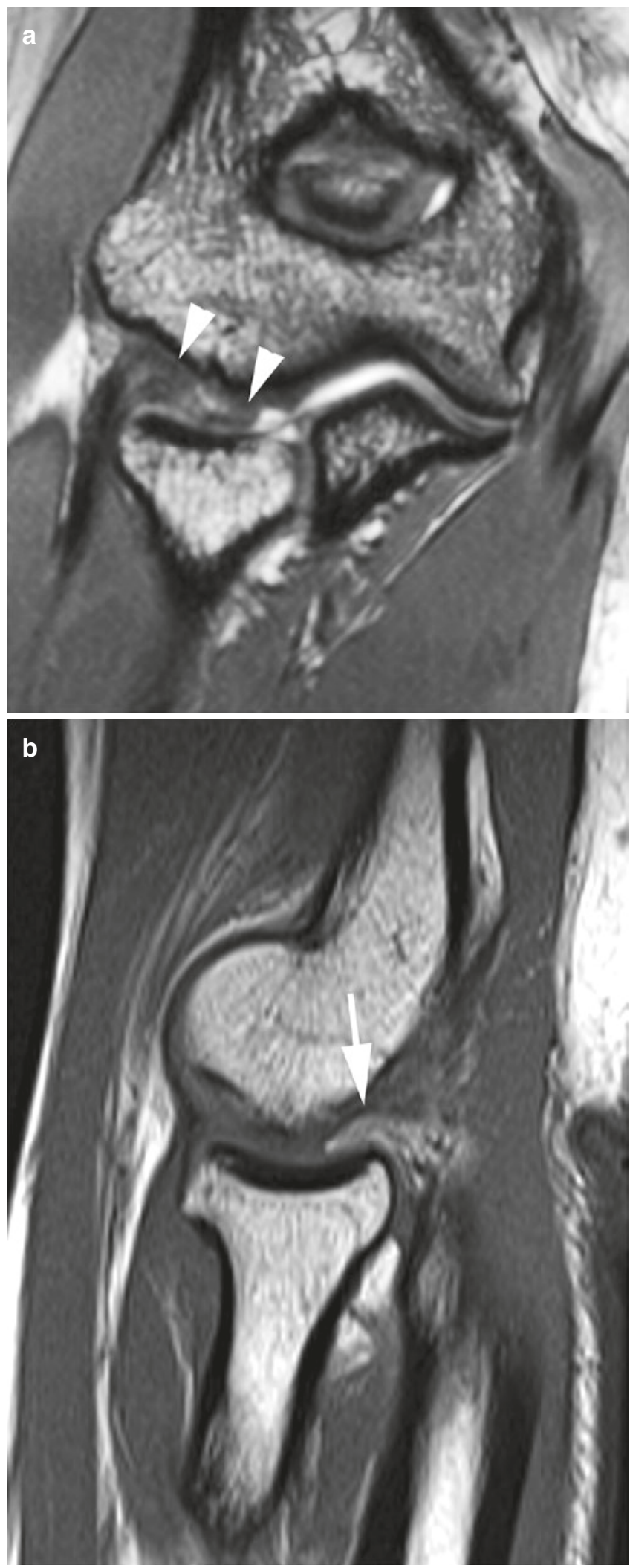

Fig. 3.20 Plica. An 18-year-old gymnast with pain in the region of the radiocapitellar joint. (a) Coronal gradient echo $\mathrm{T} 2 *$-weighted image demonstrates a prominent radiocapitellar plica (arrowheads) which is also shown to be thickened and extending into the posterior joint on a corresponding sagittal T1-weighted image (b) (arrow) 


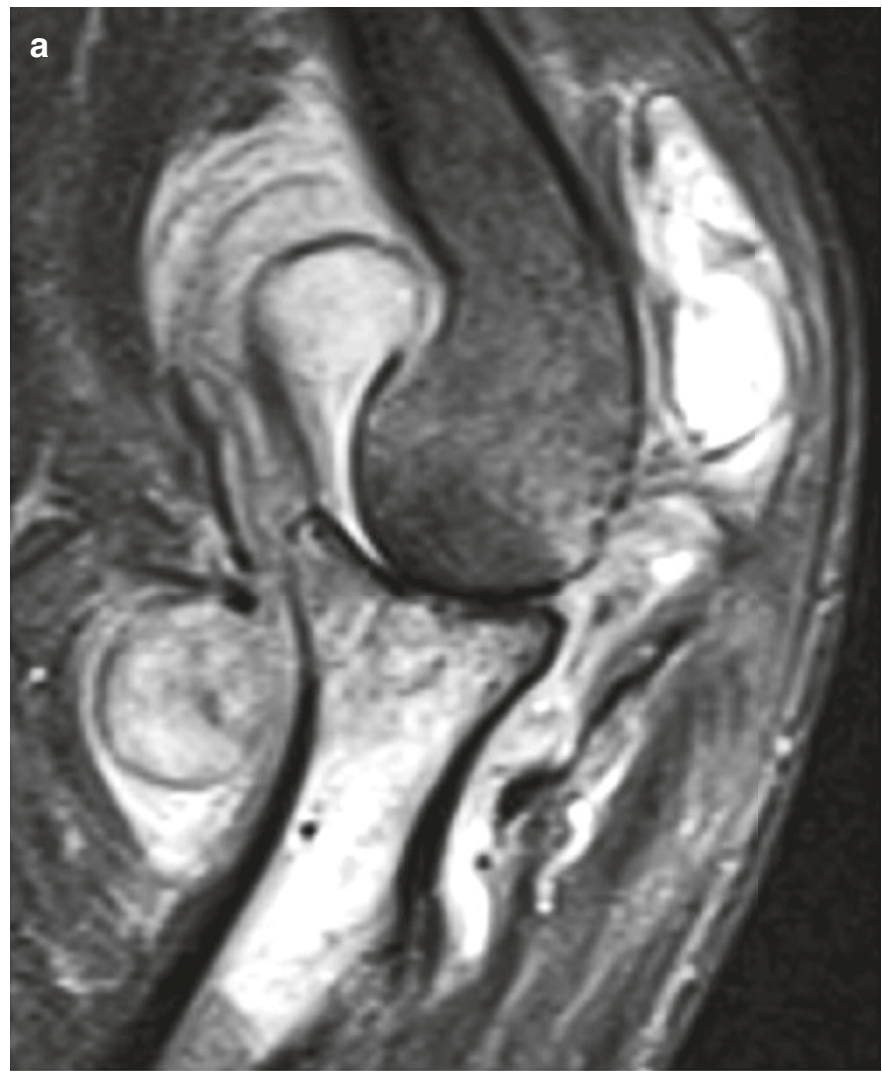

Fig. 3.21 Inflammatory synovitis. A 49-year-old female with a prior history of Lyme arthritis who developed an unspecified inflammatory polyarthropathy. (a) Sagittal fat-saturated T2-weighted image shows fluid and marked synovial hypertrophy distending the elbow joint, complete loss of articular cartilage, and prominent marrow edema in the

\subsubsection{Infectious Synovial Processes}

Septic arthritis of the upper extremity is uncommon and often occurs in patients with existing comorbidities. The nonspecific nature of imaging findings emphasizes the need for accurate correlation with clinical and laboratory findings as well as joint aspiration when there is high clinical suspicion. Any destructive monoarticular process should be regarded as infection until proven otherwise.

The majority of olecranon bursitis results from an inflammatory cause with only $25 \%$ having an infectious etiology. Septic bursitis occurs due to bacterial penetration of microtrauma or hematogenous seeding. The olecranon bursal lining has been described as poorly vascularized, resulting in an increased propensity for transcutaneous spread of infection, even when no obvious wound is present [29].

\subsubsection{Noninfectious Synovial Inflammatory Processes}

Rheumatoid arthritis is among the disorders characterized by synovial inflammation. Although the elbow is not classically an initial site of involvement in rheumatoid arthritis,

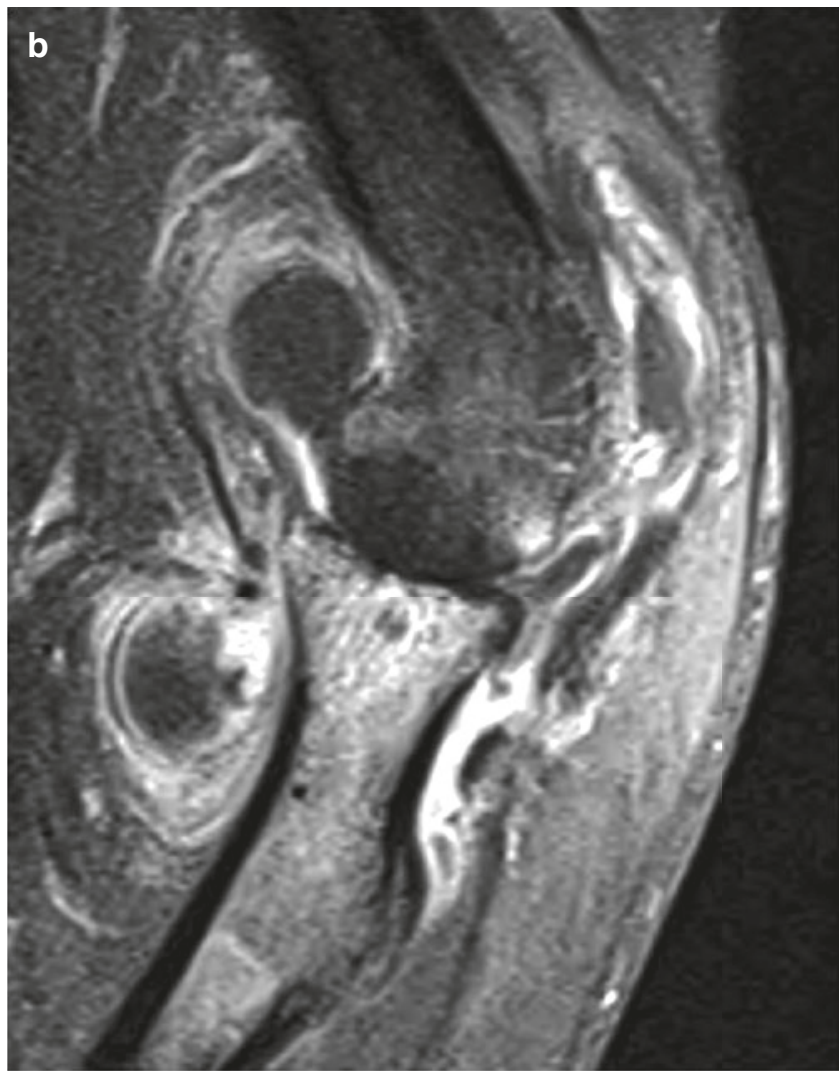

proximal radius. (b) Corresponding sagittal fat-saturated T1-weighted image after intravenous gadolinium administration provides better delineation between low signal intensity fluid and enhancing synovium. Rheumatoid arthritis or a septic joint could demonstrate a similar MR appearance

characteristic findings of synovial hypertrophy, altered marrow signal, and erosive changes are present on MRI. When it involves the olecranon bursa, inflammation with nodular involvement may be present. Gout is another rheumatologic disorder in which bursal involvement is characteristic. When sodium urate crystals are present within the bursa, low signal intensity on T1-weighted images with heterogeneous signal on fluid-sensitive sequences is noted. Radiographic correlation can be helpful to identify the cloud-like increased density seen with tophus formation. Seronegative spondyloarthropathies and calcium pyrophosphate dihydrate crystal deposition disease show MR findings of nonspecific synovial inflammation.

Pigmented villonodular synovitis (PVNS) is a rare, benign synovial proliferative process. It is an idiopathic process that most commonly affects the knee. While relatively uncommon in the elbow, the MR findings are quite characteristic. Clumps of hemosiderin-laden macrophage deposits demonstrate low signal on T1- and T2-weighted images. Gradient echo imaging enhances the magnetic susceptibility effects of the hemosiderin deposition. Joint effusion, osseous erosion, and extraarticular spread may also be seen [30]. 


\section{Key Point}

- Synovial inflammatory processes demonstrate nonspecific MR findings. In some entities, signal characteristics allow more specific diagnostic considerations. Correlation with other imaging studies, clinical presentation, and laboratory findings are important for accurate diagnosis.

\subsection{Concluding Remarks}

The elbow is a complex articulation from an anatomic and functional perspective. MR imaging has emerged as the primary tool for non-invasive evaluation of osseous and soft tissue structures, providing excellent resolution and contrast. Coupled with an understanding of disease process and mechanism of injury, MR imaging is a powerful tool for the diagnosis and characterization of disease.

\section{Take Home Messages}

- MR imaging of the elbow presents technical and diagnostic challenges that can be overcome through protocol optimization.

- Detailed understanding of anatomy and mechanism of injury is crucial for accurate diagnosis and characterization of traumatic pathology.

- Familiarity with the MR imaging appearance of osseous and soft tissue anatomy, as well as variations in anatomy, serves as the basis for improving diagnostic performance.

\section{References}

1. Johnson D, Stevens KJ, Riley G, Shapiro L, Yoshioka H, Gold GE. Approach to MR imaging of the elbow and wrist: technical aspects and innovation. Magn Reson Imaging Clin N Am. 2015;23:355-66.

2. Acosta Batlle J, Cerezal L, Márquez MV, et al. MRI of the normal elbow and common pathologic conditions. Radiographics. 2020;40:468-9.

3. Magee T. Accuracy of 3-T MR arthrography versus conventional 3-T MRI of elbow tendons and ligaments compared with surgery. Am J Roentgenol. 2015;204(1):W70-5.

4. Andreisek G, Froehlich JM, Hodler J, et al. Direct MR arthrography at 1.5 and 3.0 T: signal dependence on gadolinium and iodine concentrations—phantom study. Radiology 2008. 2008;247(3):706-16.

5. Tomsick SD, Petersen BD. Normal anatomy and anatomical variants of the elbow. Semin Musculoskelet Radiol. 2010;14:379-3.

6. Major NM, Crawford ST. Elbow effusions in trauma in adults and children: is there an occult fracture? Am J Roentgenol. 2002;178:413-8.

7. Rosenberg ZS, Blutreich SI, Schweitzer ME, Zember JS, Fillmor K. MRI features of posterior capitellar impaction injuries. Am J Roentgenol. 2008;190:435-41.

8. Smith SR, Patel NK, White AC, Hadley CJ, Dodson CC. Stress fractures of the elbow in the throwing athlete: a systematic review. Orthop J Sports Med. 2018;6:1-8.

9. Ruchelsman DE, Hall MP, Youm T. Osteochondritis dissecans of the capitellum: current concepts. J Am Acad Orthop Surg. 2010;18:557-67.

10. Marshall KW, Marshall DL, Busch MT, Williams JP. Osteochondral lesions of the humeral trochlea in the young athlete. Skelet Radiol. 2009;38:479-91.

11. Jans LBO, Ditchfield M, Anna G, Jaremko JL, Verstraete KL. MR imaging findings and MR criteria for instability in osteochondritis dissecans of the elbow in children. Eur J Radiol. 2012;81:1306-10.

12. Acosta Batlle J, Cerezal L, López Parra MD, et al. The elbow: review of anatomy and common collateral ligament complex pathology using MRI. Insights Imaging. 2019;10:43. https://doi. org/10.1186/s13244-019-0725-7.

13. Anderson MW, Alford BA. Overhead throwing injuries of the shoulder and elbow. Radiol Clin N Am. 2010;48:1137-54.

14. Joyner PW, Bruce J, Hess R, Mates A, Mills FB, Andrews JR. Magnetic resonance imaging-based classification for ulnar collateral ligament injuries of the elbow. J Shoulder Elb Surg. 2016;25:L1710-6.

15. Wei AS, Khana S, Limpisvasti O, et al. Clinical and magnetic resonance imaging findings associated with little league elbow. J Pediatr Orthop. 2010;30:715-9.

16. Kheterpal AB, Bredella MA. Overuse injuries of the elbow. Radiol Clin N Am. 2019;57:931-42.

17. Potter HG, Weiland AJ, Schatz JA, Paletta GA, Hotchkiss RN. Posterolateral rotatory instability of the elbow: usefulness of MR imaging in diagnosis. Radiology. 1997;204:185-9.

18. Kani KK, Chew FS. Terrible triad injuries of the elbow. Emerg Radiol. 2019;26:341-7.

19. Chung CB, Chew FS, Steinbach L. MR imaging of tendon abnormalities of the elbow. Magn Reson Imaging Clin N Am. 2004;12:233-45.

20. Kwak AH, Lee S-J, Jeong HS, Do MU, Kuen TS. Subtle elbow instability associated with lateral epicondylitis. BMC Musculoskeletal Disord. 2018;19:136. https://doi.org/10.1186/s12891-2069-8.

21. Quach T, Jazayeri R, Sherman OH, Rosen JE. Distal biceps tendon injuries current treatment options. Bull NYU Hosp Jt Dis. 2012;68:103-11.

22. Keener JD, Sethi PM. Distal triceps tendon injuries. Hand Clin. 2015;31:641-50.

23. Andreisek G, Crook DW, Burg D, Marincek B, Weishaupt D. peripheral neuropathies of the median, radial, and ulnar nerves: MR imaging features. Radio Graph. 2006;26:1267-87.

24. Husarik DB, Saupe N, Pfirrmann CW, Jost B, Hodler J, Zanetti M. Elbow nerves: MR findings in 60 asymptomatic subjects-normal anatomy, variants, and pitfalls. Radiology. 2009;252(1):148-56.

25. Floemer F, Morrison WB, Bongartz G, Ledermann HP. MRI characteristics of olecranon bursitis. Am J Roentgenol. 2004;183:29-34.

26. Skaf AY, Boutin RD, Dantas RWM, et al. Bicipitoradial bursitis: MR imaging findings in eight patients and anatomic data from con- 
trast material opacification of bursae followed by routine radiography and MR imaging in cadavers. Radiology. 1999;212:111-6.

27. Cerezal L, Rodriguez-Sammartino M, Canga A, et al. Elbow synovial fold syndrome. Am J Roentgenol. 2013;201:W88-96.

28. Jbara M, Patnana M, Kazmi F, Beltran J. MR imaging: arthropathies and infectious conditions of the elbow, wrist, and hand. Radiol Clin N Am. 2006;44:625-42.
29. Blackwell JR, Hay BA, Bolt AM, Hay SM. Olecranon bursitis: a systematic overview. Shoulder Elbow. 2014;6:182-90.

30. Lu H, Chen Q, Shen H. Pigmented villonodular synovitis of the elbow with radial, median and ulnar nerve compression. Int J Clin Exp Pathol. 2015;8:14045-9.

Open Access This chapter is licensed under the terms of the Creative Commons Attribution 4.0 International License (http://creativecommons. $\mathrm{org} / \mathrm{licenses} / \mathrm{by} / 4.0 /$ ), which permits use, sharing, adaptation, distribution and reproduction in any medium or format, as long as you give appropriate credit to the original author(s) and the source, provide a link to the Creative Commons license and indicate if changes were made.

The images or other third party material in this chapter are included in the chapter's Creative Commons license, unless indicated otherwise in a credit line to the material. If material is not included in the chapter's Creative Commons license and your intended use is not permitted by statutory regulation or exceeds the permitted use, you will need to obtain permission directly from the copyright holder. 